\title{
Acute fulminant myocarditis following influenza vaccination requiring extracorporeal membrane oxygenation
}

\author{
Youn-Jung Kim, Jun-II Bae, Seung Mok Ryoo, Won Young Kim \\ Department of Emergency Medicine, Asan Medical Center, University of Ulsan College of Medicine, Seoul, Korea
}

The inactivated influenza vaccination is generally safe with mostly mild side effects. We report a rare but fatal adverse event following influenza vaccination. A previously healthy 27 -yearold woman who received the influenza vaccination 3 days before presenting to the emergency department had rapidly aggravating dyspnea and mental deterioration. She was diagnosed as having acute fulminant myocarditis with refractory cardiogenic shock, which was successfully managed with veno-arterial extracorporeal membrane oxygenation. The cardiac function of the patient recovered in 3 weeks.

Key Words: adverse event; cardiogenic shock; extracorporeal membrane oxygenation; influenza vaccines; myocarditis

Influenza is an acute respiratory illness caused by the influenza A or B virus, which occurs in distinct annual outbreaks. Influenza causes highly variable clinical manifestations ranging from fever, general myalgia, fatigue, cough, and sore throat to neurologic complications, respiratory failure, shock, and death. The morbidity and mortality caused by influenza are substantial in the general population. Patients who are at high risk of serious complications from influenza include those aged 65 years or older, children aged between 6 and 59 months, those with chronic medical disorders of the respiratory, cardiovascular, hepatic and renal systems, and immunocompromised patients [1]. In Korea, the Korea Centers for Disease Control and Prevention [1] recommends annual influenza vaccinations for these high-risk groups due to their effectiveness in preventing influenza and reducing the burden of complications.

The inactivated influenza vaccination is usually well-tolerated and the side effects are mostly benign local or general reactions, including pain at the injection site, fever, or myalgia [2]. Serious adverse events have been very rarely reported and there have been few case reports of cardiac complications such as pericarditis, myocarditis, and stress-induced cardiomyopathy [3-5]. However, the mechanism of cardiac complications following influenza vaccination is not fully elucidated due its rare incidence. Herein, we report a case of acute fulminant myocarditis occurring after quadrivalent inactivated influenza vaccination (GC Flu Quadrivalent; Green Cross, Yongin, Korea), rescued by extracorporeal membrane oxygenation (ECMO).

\section{Case Report}

Received: January 25, 2017 Revised: April 29, 2017

Accepted: May 2, 2017

Corresponding author Won Young Kim Department of Emergency Medicine, Asan Medical Center, University of Ulsan College of Medicine, 88 Olympic-ro 43-gil, Songpa-gu, Seoul 05505, Korea

Tel: +82-2-3010-3350

Fax: +82-2-3010-3360

E-mail:wonpia73@naver.com

Copyright $\odot 2019$ The Korean Society of Critical Care Medicine

This is an Open Access article distributed under the terms of Creative Attributions Non-Commercial License (http:// creativecommons.org/li-censes/by-nc/4.0/) which permits unrestricted noncommercial use, distribution, and reproduction in any medium, provided the original work is properly cited. 


\section{CASE REPORT}

The study was performed according to the Helsinki Declaration and approved by the Institutional Review Board of Asan Medical Center (IRB No. 2017-0213), and the informed consent was waived.

A 27-year-old woman without any previous medical history presented to the Emergency Department at Asan Medical Center, Seoul, Korea with a headache, nausea, vomiting, low back pain, and fatigue. Three days before presentation, she received an intramuscular injection against influenza. A quadrivalent inactivated influenza vaccination (GC Flu Quadrivalent, Green Cross) was administered, containing $15 \mu \mathrm{g}$ hemagglutinin each of the same two A strains per $0.5 \mathrm{ml}$ dose: an A/Christchurch/16/2010 NIB-74xp (H1N1), A/Hongkong/4801/ 2014 NYMC X-263B (H3N2), B/Brisbane/60/2008, and B/Phuket/3073/2013. Two days later, she developed low back pain, and after ingestion of a nonsteroidal anti-inflammatory drug for pain relief, headache, nausea, and vomiting also developed.

At initial presentation, her vital signs were as follows: blood pressure, 145/101 mmHg; pulse rate, 116 beats per minute; respiratory rate, 20 breaths per minute; body temperature, $37.4^{\circ} \mathrm{C}$; and oxygen saturation, $95 \%$ on room air (Figure 1 ). General physical examination revealed unremarkable findings and upon neurological examination; she had no sign of meningismus. However, she developed dyspnea and a cough with pink, frothy sputum after 4 hours of waiting for the laboratory results. Her pulse and respiratory rates had elevated to 169 beats per minute and 30 breaths per minute, respectively. Her oxygen saturation ranged from $83 \%$ to $95 \%$ despite the constant oxygen supply via an oxygen mask with a reservoir bag and a flow rate of $15 \mathrm{~L} / \mathrm{min}$. A chest X-ray also demonstrated significant changes over the 4-hour period, during which she went from having a normal chest to having diffuse ground glass opacities in both lung fields, which suggested pulmonary edema or impending acute respiratory distress syndrome (Figure 2A). Her electrocardiogram demonstrated sinus tachycardia without STsegment changes.

Her initial laboratory findings are presented in Table 1. Cardiac troponin I was elevated at $25.140 \mathrm{ng} / \mathrm{ml}$, as was creatine kinase-MB at $54.2 \mathrm{ng} / \mathrm{ml}$ and creatine kinase at $628 \mathrm{IU} / \mathrm{L}$. Brain natriuretic peptide was $1,803 \mathrm{pg} / \mathrm{ml}$ (normal $<100 \mathrm{pg} / \mathrm{ml}$ ). Blood and sputum cultures were negative and a respiratory polymerase chain reaction panel to detect common respiratory pathogens also showed unremarkable findings. Bedside transthoracic echocardiogram demonstrated biventricular akinesia of the mid to apical walls, implying biventricular dysfunction. The left ventricular ejection fraction (LVEF), which was estimated using Simpson's biplane method, was $15 \%$. Mild mitral regurgitation was also detectable via color Doppler analysis.

Respiratory distress and mental deterioration were ob-

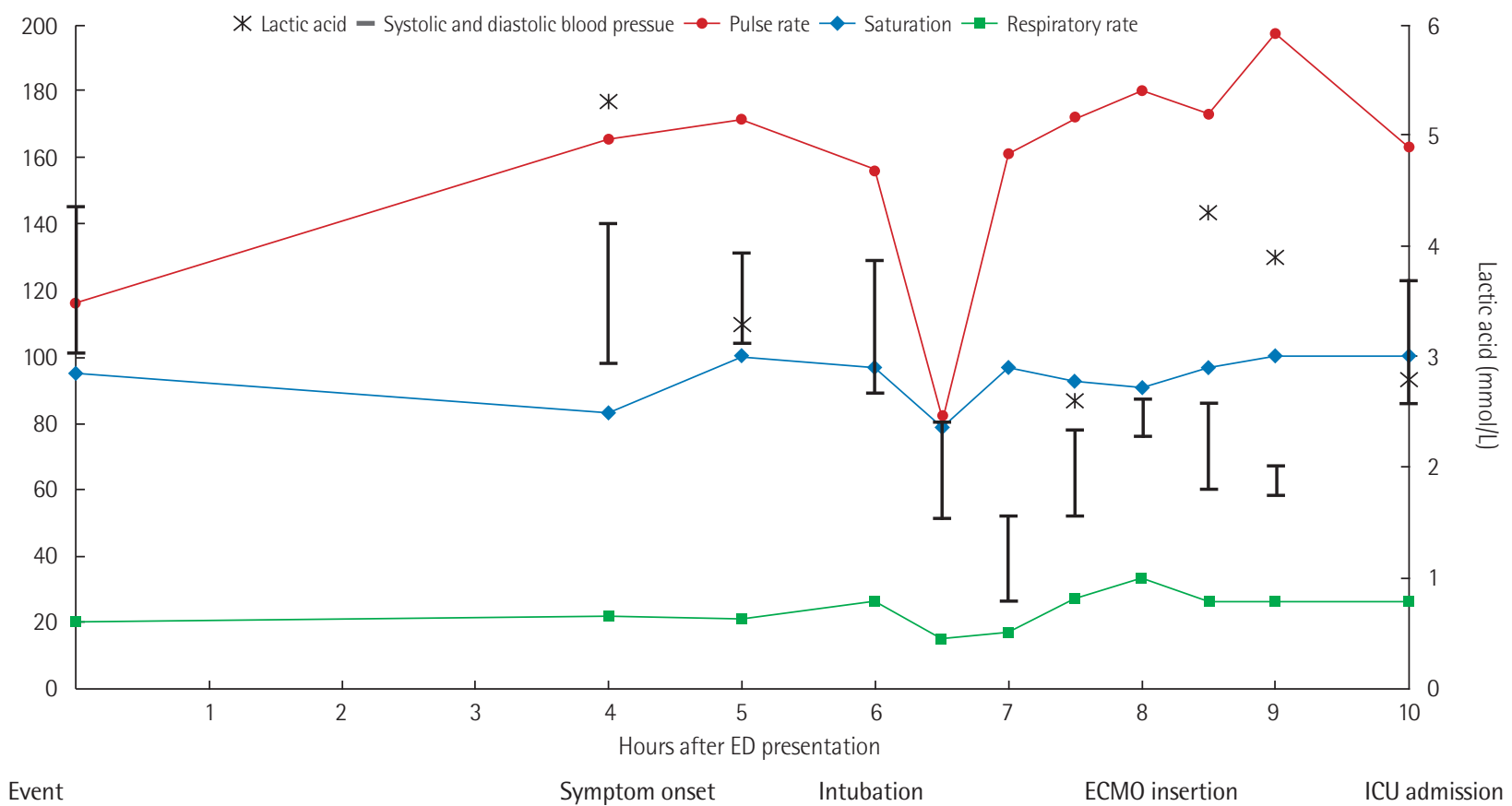

Figure 1. Serial changes of vital signs and lactic acid level for 10 hours after emergency department (ED) presentation. ECM0: extracorporeal membrane oxygenation; ICU: intensive care unit. 

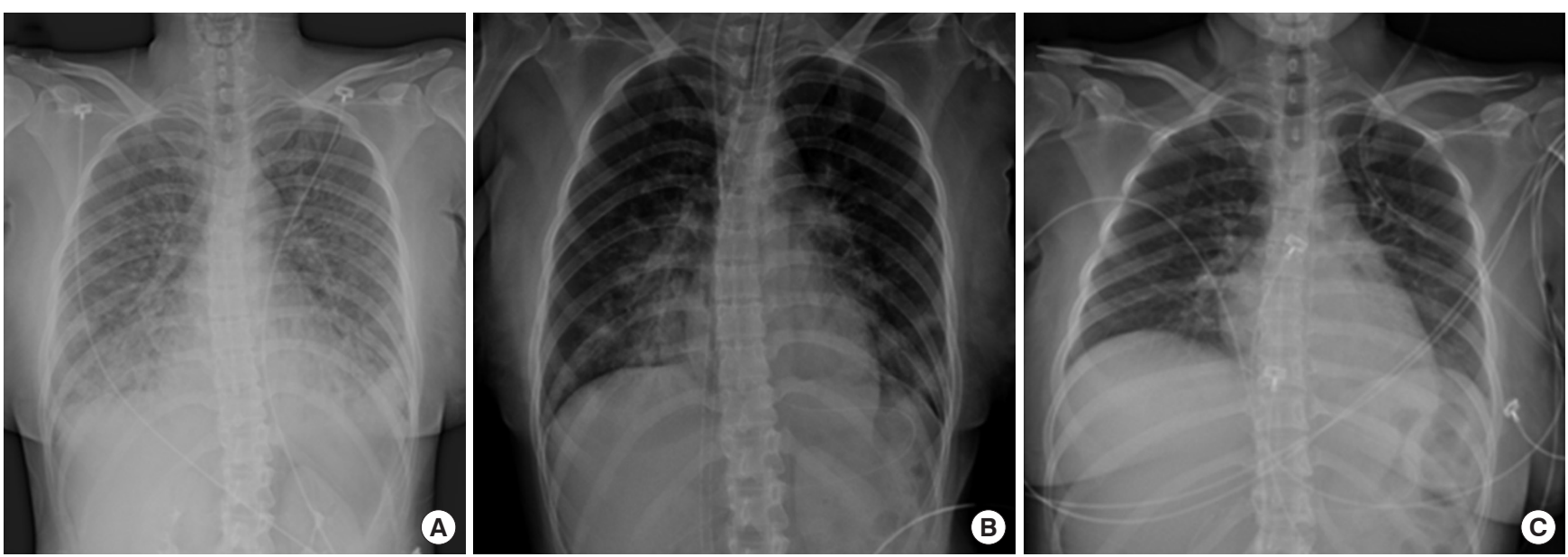

Figure 2. Serial chest X-rays during hospitalization. (A) Chest X-ray showed diffuse ground glass opacities on both lung fields at 4 hours after emergency department presentation. (B) Chest X-ray after insertion of extracorporeal membrane oxygenation and intubation at emergency department. (C) Chest $\mathrm{X}$-ray after recovery on the 9 th day of hospitalization.

Table 1. Serial changes of laboratory findings

\begin{tabular}{|c|c|c|c|c|c|}
\hline \multirow{2}{*}{ Variable } & \multicolumn{5}{|c|}{ Hospitalization day } \\
\hline & $\# 1$ & \#2 & \#3 & $\# 4$ & $\# 5$ \\
\hline White blood cell $(/ \mu l)$ & 27,300 & 23,500 & 16,200 & 15,900 & 13,200 \\
\hline C-reactive protein (mg/dl) & 4.27 & 14.22 & 24.03 & 18.66 & 9.97 \\
\hline Creatine kinase (IU/L) & 628 & 869 & 18,220 & 21,085 & 15,350 \\
\hline Creatine kinase-MB (ng/ml) & 54.2 & 25.5 & 74.6 & 50.0 & 56.2 \\
\hline Troponin I (ng/ml) & 25.14 & 16.53 & 12.45 & 6.03 & 2.71 \\
\hline
\end{tabular}
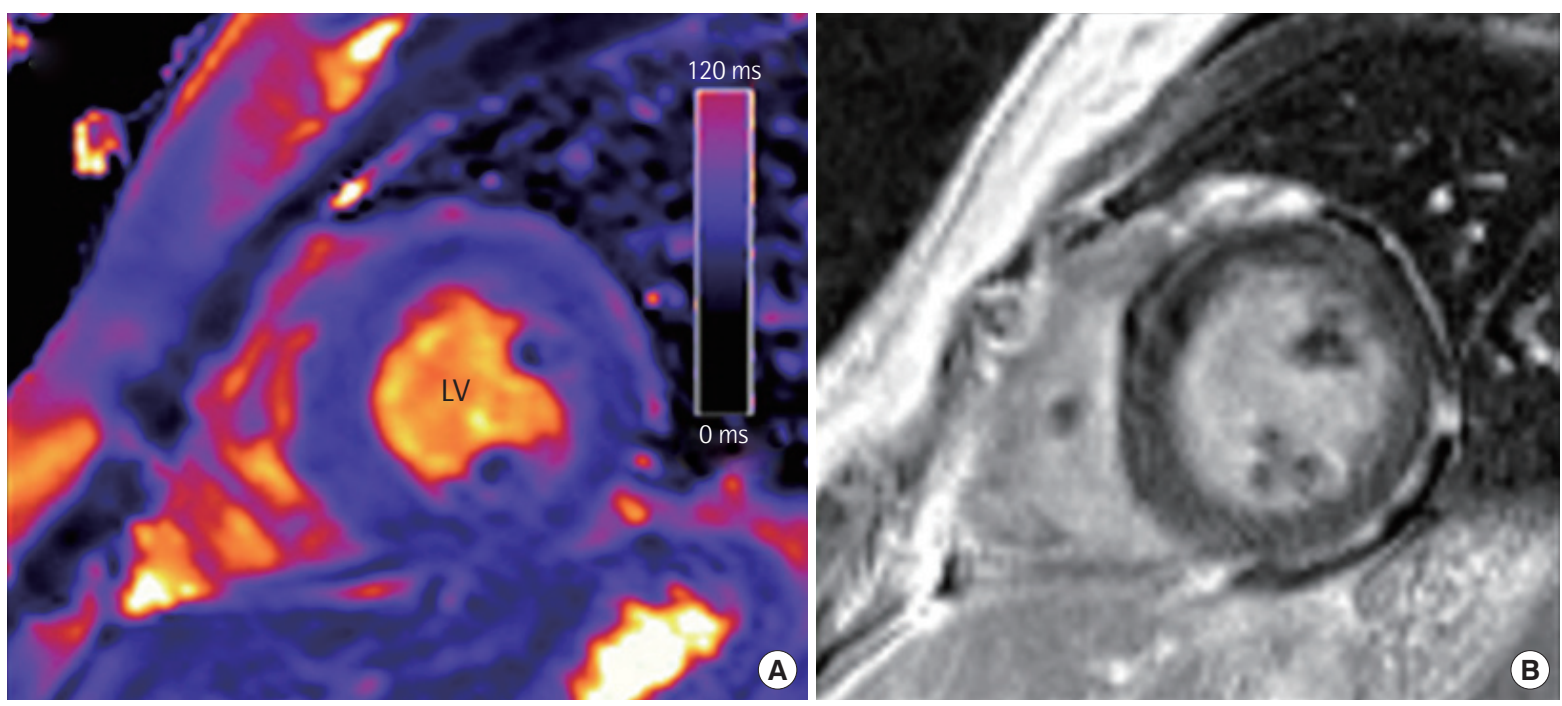

Figure 3. Representative cardiovascular magnetic resonance findings in a patient with acute myocarditis. (A) Short-axis T2 map revealed an increased global myocardial T2 value of 68-72 ms in this patient. (B) Late gadolinium enhancement shows mild diffuse midwall enhancement in the left ventricular (LV) myocardium.

served soon after bedside echocardiography, therefore endotracheal intubation was performed. Despite ventilator care and vasopressor infusion, her blood pressure still remained below 60 to $70 \mathrm{mmHg}$ and tissue hypoperfusion, which was reflective of elevated lactic acid levels, was observed. To save the patient from refractory cardiogenic shock, emergency ve- 
no-arterial ECMO was performed in the emergency department (Figure 2B).

She was admitted to the emergency intensive care unit, where she gradually recovered. She was successfully weaned from ECMO on the 5th day of hospitalization, and from the mechanical ventilator on the 9th day (Figure 2C), when follow-up echocardiography showed improved left ventricular dysfunction (LVEF, 48\%). Cardiovascular magnetic resonance imaging was performed on the 14th day of hospitalization, which demonstrated slightly increased global myocardial T2 values without delayed enhancement in the left ventricular myocardium, indicating an improved status of acute myocarditis (Figure 3). Follow-up echocardiography on the 21st day confirmed the normalized left ventricular function.

\section{DISCUSSION}

We have reported a case of acute fulminant myocarditis in a previously healthy young female following the administration of a quadrivalent inactivated influenza vaccination. Since Streifler et al. [6] described a patient with recurrent pericarditis after influenza vaccination in 1981, a very limited number of patients with cardiac complications following influenza vaccination have been reported. It is still controversial whether myocarditis was causally related to influenza vaccination or merely a serendipitous occurrence. To the best of our knowledge, only one study about adverse events using the Vaccine Adverse Event Reporting System database reported a fatal case of myocarditis in children between 6 to 23 months after administration of a trivalent inactivated influenza vaccine, but the authors concluded the causal relationship between influenza vaccination and development of myocarditis was not certain [7].

It is not possible to prove the hypothetical mechanism of the development of myocarditis after influenza vaccination due to its rarity as well as the associated ethical problems, but one hypothesis suggests that the immune response to compounds in the influenza vaccination and its specific inflammatory cascades may play a major role in the development of cardiovascular compromise $[4,8]$. It is notable that our patient received an inactivated quadrivalent influenza vaccine without adjuvant (GC Flu Quadrivalent, Green Cross). Previous case [4] reported that their patients received an inactivated trivalent influenza vaccine with MF59 adjuvant (Fluad, Novartis). This implies the possibility that other mechanisms also contribute to the development of cardiovascular compromise after influenza vaccination more so than the autoim- mune/inflammatory syndrome induced by adjuvants.

The true incidence of myocarditis after influenza vaccination may be underestimated because of the subclinical and nonspecific clinical manifestations of myocarditis. A prospective study about the incidence of cardiovascular adverse events after smallpox and influenza vaccination revealed that the rate of clinical or subclinical myocarditis and pericarditis after smallpox vaccination was approximately $1: 5,500$, despite the fact that the rate of troponin elevation was 1:200 [9]. Similarly, $2.6 \%$ of the influenza vaccine cohort showed new onset cardiac symptoms, such as chest pain, dyspnea, and palpitation, but no case of clinical or subclinical myocarditis and pericarditis [9].

In this study, we have reported a very rare and serious adverse event following influenza vaccination. In light of this report, influenza vaccination should not be limited; however, physicians should be aware of the possibility of serious cardiovascular complications after influenza vaccination and thus be prepared to provide high-quality intensive care if necessary.

\section{CONFLICT OF INTEREST}

No potential conflict of interest relevant to this article was reported.

\section{ORCID}

Youn-Jung Kim https://orcid.org/0000-0003-1385-5836

Seung Mok Ryoo https://orcid.org/0000-0002-2436-3311

Won Young Kim https://orcid.org/0000-0002-6904-5966

\section{AUTHOR CONTRIBUTIONS}

Conceptualization: WYK. Data curation: YJK. Project administration: WYK. Visualization: YJK. Writing - original draft: YJK. Writing - review \& editing: all authors.

\section{REFERENCES}

1. Korea Centers for Disease Control and Prevention. Korea Centers for Disease Control and Prevention (KCDC) web [Internet]. Cheongju: Korea Centers for Disease Control and Prevention; 2017 [cited 2017 Oct 19]. Available from: http://cdc.go.kr/ CDC/mobile/intro/CdcKrIntro0505.jsp?menuIds=HOME001MNU1154-MNU2557-MNU2487\&cid=76303.

2. Nichol KL, Margolis KL, Lind A, Murdoch M, Mc-Fadden R, Hauge M, et al. Side effects associated with influenza vaccination in healthy working adults: a randomized, placebo- 
controlled trial. Arch Intern Med 1996;156:1546-50.

3. de Meester A, Luwaert R, Chaudron JM. Symptomatic pericarditis after influenza vaccination: report of two cases. Chest 2000;117:1803-5.

4. Cheng MP, Kozoriz MG, Ahmadi AA, Kelsall J, Paquette K, Onrot JM. Post-vaccination myositis and myocarditis in a previously healthy male. Allergy Asthma Clin Immunol 2016;12:6.

5. Singh K, Marinelli T, Horowitz JD. Takotsubo cardiomyopathy after anti-influenza vaccination: catecholaminergic effects of immune system. Am J Emerg Med 2013;31:1627.e1-4.

6. Streifler JJ, Dux S, Garty M, Rosenfeld JB. Recurrent pericarditis: a rare complication of influenza vaccination. Br Med J (Clin Res Ed) 1981;283:526-7.
7. Rosenberg M, Sparks R, McMahon A, Iskander J, Campbell JD, Edwards KM. Serious adverse events rarely reported after trivalent inactivated influenza vaccine (TIV) in children 6-23 months of age. Vaccine 2009;27:4278-83.

8. Lanza GA, Barone L, Scalone G, Pitocco D, Sgueglia GA, Mollo $\mathrm{R}$, et al. Inflammation-related effects of adjuvant influenza A vaccination on platelet activation and cardiac autonomic function. J Intern Med 2011;269:118-25.

9. Engler RJ, Nelson MR, Collins LC Jr, Spooner C, Hemann BA, Gibbs BT, et al. A prospective study of the incidence of myocarditis/pericarditis and new onset cardiac symptoms following smallpox and influenza vaccination. PLoS One 2015;10: e0118283. 